\title{
Video Article \\ Vinyl Chloride and High-Fat Diet as a Model of Environment and Obesity Interaction
}

\author{
Anna L. Lang ${ }^{1,2}$, William T. Goldsmith ${ }^{3,4}$, Regina D. Schnegelberger ${ }^{5,6}$, Gavin E. Arteel ${ }^{6,7}$, Juliane I. Beier ${ }^{6,7}$ \\ ${ }^{1}$ Department of Pharmacology and Toxicology, University of Louisville \\ ${ }^{2}$ Hepatobiology and Toxicology Program, University of Louisville \\ ${ }^{3}$ Department of Physiology and Pharmacology, West Virginia University \\ ${ }^{4}$ Center for Inhalation Toxicology, West Virginia University \\ ${ }^{5}$ Department of Pharmacology and Chemical Biology, University of Pittsburgh \\ ${ }^{6}$ Pittsburgh Liver Research Center, University of Pittsburgh \\ ${ }^{7}$ Department of Medicine, Division of Gastroenterology, Hepatology and Nutrition, University of Pittsburgh
}

Correspondence to: Juliane I. Beier at jibeier@pitt.edu

URL: https://www.jove.com/video/60351

DOI: doi:10.3791/60351

Keywords: Medicine, Issue 155, vinyl chloride, chloroethene, inhalation, environmental toxicants, chemicals, organochlorine, liver disease, volatile organic compounds, toxicant-associated steatohepatitis, non-alcoholic fatty liver disease

Date Published: 1/12/2020

Citation: Lang, A.L., Goldsmith, W.T., Schnegelberger, R.D., Arteel, G.E., Beier, J.I. Vinyl Chloride and High-Fat Diet as a Model of Environment and Obesity Interaction. J. Vis. Exp. (155), e60351, doi:10.3791/60351 (2020).

\section{Abstract}

Vinyl chloride (VC), an abundant environmental contaminant, causes steatohepatitis at high levels, but is considered safe at lower levels Although several studies have investigated the role of VC as a direct hepatotoxicant, the concept that VC modifies sensitivity of the liver to other factors, such as nonalcoholic fatty liver disease (NAFLD) caused by high-fat diet (HFD) is novel. This protocol describes an exposure paradigm to evaluate the effects of chronic, low-level exposure to VC. Mice are acclimated to low-fat or high-fat diet one week prior to the beginning of the inhalation exposure and remain on these diets throughout the experiment. Mice are exposed to VC (sub-OSHA level: <1 ppm) or room air in inhalation chambers for 6 hours/day, 5 days/week, for up to 12 weeks. Animals are monitored weekly for body weight gain and food consumption. This model of VC exposure causes no overt liver injury with VC inhalation alone. However, the combination of VC and HFD significantly enhances liver disease. A technical advantage of this co-exposure model is the whole-body exposure, without restraint. Moreover, the conditions more closely resemble a very common human situation of a combined exposure to VC with underlying nonalcoholic fatty liver disease and therefore support the novel hypothesis that $\mathrm{VC}$ is an environmental risk factor for the development of liver damage as a complication of obesity (i.e., NAFLD). This work challenges the paradigm that the current exposure limits of VC (occupational and environmental) are safe. The use of this model can shed new light and concern on the risks of VC exposure. This model of toxicant-induced liver injury can be used for other volatile organic compounds and to study other interactions that may impact the liver and other organ systems.

\section{Video Link}

The video component of this article can be found at https://www.jove.com/video/60351/

\section{Introduction}

Numerous toxicants are present in the air we breathe at very low levels. Vinyl chloride (VC) is monomeric gas used by industry to create polyvinyl chloride (PVC) plastic products ${ }^{1}$. It is a prevalent environmental hepatotoxicant, known carcinogen, and is ranked \#4 on the ATSDR Hazardous Substance Priority List ${ }^{2}$. To better understand the toxic effects on human health and interactions with existing co-morbidities, establishing models of exposure that mimic human exposure is crucial. The primary interest of this group is to study the hepatic effects of chronic VC exposure at low concentrations. VC exerts its main effects on the liver, where it has been shown (at high concentrations) to cause steatosis, and toxicant-associated steatohepatitis (TASH) with necrosis, fibrosis, cirrhosis ${ }^{3,4}$, as well as hepatocellular carcinoma (HCC) and the otherwise extremely rare hepatic hemangiosarcoma ${ }^{5}$. TASH has likely existed in the population for decades but remained uncharacterized and underappreciated by investigators ${ }^{4,6}$. As a result of research demonstrating the direct toxicity concerns for VC exposure, the Occupational Safety and Health Administration (OSHA) lowered the acceptable exposure threshold to $1 \mathrm{ppm}$ over an $8 \mathrm{~h}$ work day ${ }^{7}$. Although the exposure threshold has been lowered, the effect of this concentration of VC on human health is unclear ${ }^{7}$. Additionally, the effect of VC exposure on existing comorbidities, such as liver disease, is largely unknown ${ }^{8}$. This knowledge gap is especially important today due to the increasing global

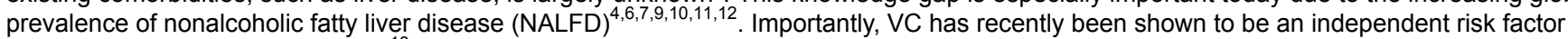
for liver disease from other causes ${ }^{13}$. The goal of this protocol was therefore to develop a relevant inhalation model for exposure to the volatile environmental toxicant, VC in the context of underlying liver injury, to mimic human exposure and identify potential, novel mechanisms of VCinduced or VC-enhanced liver injury.

The main route of exposure for many environmental toxicants and pollutants is via inhalation. Once inhaled, the compound can enter systemic circulation through the lungs, travel to the liver, and become metabolically activated by hepatic enzymes prior to being excreted ${ }^{14,15,16}$. It is often 
these active metabolites that cause toxicity and damage within the body. Previous studies by this group and others have used VC metabolites as surrogates for exposure to VC gas ${ }^{17,18}$. Other groups have used inhalation models of VC; however, extremely high exposure levels $(>50$ $\mathrm{ppm}$ ) were implemented to induce acute toxicity, severe hepatic injury, and tumor development ${ }^{19}$. Although these studies have provided crucial information and mechanisms of VC-induced carcinogenicity, they do not recapitulate the subtle effects and complex interactions with other contributing factors and therefore are less relevant to human exposure.

The VC-inhalation plus high fat diet (HFD) model described here (see Figure 1 for timeline), is the first model of chronic, low-dose VC exposure (i.e., sub-OSHA concentration), in which mice are exposed to the toxicant under conditions that mimic human exposure much more closely. Indeed, data from this model recapitulated results observed in humans exposed to VC, such as the impact on metabolic pathways ${ }^{20}$, oxidative stress and mitochondrial dysfunction ${ }^{4}$. Other mouse models of inhalation, such as head-only and nose-only models ${ }^{21}$, require that the animal be restrained, causing stress to the animal. Here, this whole-body exposure method does not require injection or unneeded stress to the animals. The animals have ad libitum access to food and water and are placed within the larger inhalation chamber for a determined number of hours per day and days per week. Moreover, the concept that $\mathrm{VC}$ modifies sensitivity to another hepatotoxicant is a novel finding, first demonstrated by this group $^{12}$ and has implications for VC exposure at concentrations well below those needed for direct hepatotoxicity.

This method of inhalation exposure can be used to mimic exposure to a variety of gaseous toxicants, including other volatile organic compounds, present in our environment. Indeed, volatile organic compounds are a large group of environmental toxicants and more prevalent in industrialized areas, resulting in certain populations being at higher risk for chronic exposure ${ }^{22}$. This protocol can be modified to suit different experimental questions. The length of time and concentration of compound administered can be varied. Although initially developed for determination of liver injury, other organ systems can and have been studied with this model ${ }^{23}$. Investigators who aim to study chronic exposures with animals, but wish to minimize animal stress, should consider using this model.

\section{Protocol}

All of the animal/VC experiments were approved by the Department of Environmental Health, the Safety Association for Assessment and Accreditation of Laboratory Animal Care and procedures were approved by the local Institutional Animal Care and Use Committee.

\section{Experimental set-up and acclimatization to purified, experimental diets}

1. Determine the total number of $\mathrm{C} 56 \mathrm{BI} / 6 \mathrm{~J}$ mice (minimally 6-8 mice per group).

NOTE: Animals of each diet group will be further sub-divided into exposure groups. Be sure to account for total number of animals needed when planning the study.

2. Identify and weigh the animals. Record these data.

3. Switch diets from regular chow to purified low-fat (LFD) or high-fat diet (HFD) one week prior to start of the inhalation experiments to acclimate the mice to the new diets (see Figure 1 for timeline).

4. Provide food and water ad libitum. Monitor food consumption by weighing and recording the food to be given per cage, and weighing and recording the remainder of the food at each feeding day. If housing 4 mice per cage, provide $\sim 50 \mathrm{~g}$ of food twice per week. If housing 5 mice per cage, provide $\sim 60 \mathrm{~g}$ of food twice per week.

NOTE: During the feeding of the purified diets, the amount of food should be checked every day to ensure the mice have sufficient pellets. If there are insufficient pellets the mice tend to 'hoard' food and increase the intake. Moreover, especially the HFD tends to crumble much more than the LFD, causing a similar effect.

5. Monitor animals throughout the experiment to ensure animal health is maintained.

NOTE: Weekly weight gain and food consumption, along with metabolic monitoring can be done to provide an index of overall animal health.

\section{Vinyl chloride inhalation exposure system}

NOTE: There are multiple inhalation exposure systems commercially available, ranging from 'nose-only' to 'whole-body' exposure and manual to automated systems. Data previously published by this group was derived from a whole-body manual system ${ }^{12,23,24}$. A diagram describing the automated inhalation exposure system is shown in Figure 2.

1. Ensure that the diluent air in both the experimental and control chambers is high-efficiency particulate air (HEPA) and activated carbon filtered, dried and pressure regulated before entering their respective flow measurement devices (mass flow controller [MFC]-experimental chamber, rotameter-control chamber).

NOTE: In the control chamber, the rotameter regulates the airflow to the mice. The air enters the top of the chamber, passes by the mice, then is exhausted under the mice and passed through a HEPA filter before entering the chemical hood. Temperature and relative humidity $(\mathrm{RH})$ are measured within the chamber. In the experimental chamber, the diluent air is mixed with air from a VC tank. Both flows are regulated with MFCs. The ratio of the two mixtures determines the concentration of VC in the experimental chamber. The VC enters the top of the exposure chamber through a disperser with seven jets that point in different directions. The VC passes by the mice and is then exhausted through 12 separate ports that are positioned underneath the cage rack. This chamber design has been shown to provide homogenous toxicant concentrations previously ${ }^{25}$.

2. Ensure that the pressure, temperature and $\mathrm{RH}$ are monitored from within the experimental and the control chambers.

3. Confirm that the chamber exhaust is passed through a HEPA filter, a $\mathrm{CO}_{2}$ probe, and an activated carbon filter before entering the exhaust area of the chemical hood and that the $\mathrm{CO}_{2}$ level is monitored to ensure the mice are receiving acceptable ventilation.

4. Use the custom software to change, monitor and record environmental variables during inhalation exposures.

NOTE: If a manual system is used, the variables described in steps $2.1-2.4$ should be monitored and calibrated, when necessary regularly throughout the exposure period. 


\section{Pre-exposure set-up}

1. Turn off all airflows in the experimental and control chambers for technician safety.

2. For each chamber, open the chamber door and place absorbent bedding material (absorbent side up) on top of the excreta pan. Wet the absorbent material to provide a comfortable humidity level $(40-60 \% \mathrm{RH})$ throughout the exposure period.

3. Set the desired exposure level of VC in the chamber. For sub-OSHA limit concentrations use $0.85 \mathrm{ppm}$ of VC. Use either the softwaremanaged, detector-based feedback control of VC delivery to the chamber or use manual adjustments to the system.

NOTE: The latter approach requires knowledge of the chamber volume, chamber refresh rate, airflow and delivery rate of the VC gas from the stock supply; these calculations must subsequently be validated and calibrated by measurements of VC concentrations in the chamber at steady state ${ }^{12,24}$. The most common technique for measuring VC in the chamber is via gas chromatographic analysis of sample air ${ }^{12,24}$. The advantages of the software-driven approach regarding accuracy and precision of VC delivery are clear. However, it has been shown that the manual approach is also accurate and consistent ${ }^{12,24}$.

CAUTION: VC is a known toxicant and carcinogen at high levels. Exercise proper personal protective equipment and handling of the gas while turning on and off the chambers.

\section{Exposure cage and animal preparation}

1. Remove the mice from their housing chambers and place them into the individual cages of the inhalation chamber cage rack (one cage rack for the control mice, one for the exposed mice). Randomize each mouse's placement within the cage rack daily to ensure that each mouse is exposed homogenously within the exposure chamber. Mark each animal's number and cage placement position in the laboratory notebook.

2. Place each cage rack into its respective chamber and close the chamber doors.

\section{Conducting an exposure}

1. Ensure that the valve for the $\mathrm{VC}$ gas tank is in the open position. Ensure that the diluent flow for the experimental chamber is set to $25 \mathrm{~L} / \mathrm{min}$.

2. Start the diluent flow in the experimental chamber. Ensure the rotameter on the control chamber is set to $25 \mathrm{~L} / \mathrm{min}$.

3. Ensure that all sensors (flows, temperature, humidity, chamber pressure, $\mathrm{CO}_{2}$ level) are working correctly and displaying expected results in both the experimental and control chambers.

NOTE: The VC flow is calculated and set based on the diluent flow and desired VC concentration.

4. Ensure that throughout the exposure, in the experimental chamber, the exposure time, diluent flow, VC flow, temperature, humidity, chamber pressure, $\mathrm{CO}_{2}$ level, and theoretical VC concentration are displayed, graphed and recorded. Confirm that the temperature and humidity for the control chamber are also displayed, graphed and recorded.

NOTE: If a manual system is used, VC flow should be checked and adjusted, when necessary, throughout the exposure period.

5. If any problems occur during the exposure, set the $\mathrm{VC}$ flow to zero and increase the diluent flow to its maximum value to quickly purge the chamber.

6. Once the exposure duration (i.e., $6 \mathrm{~h} /$ day) has been reached, the software automatically turns off the VC flow. The 15 min safety timer then begins for the time after duration for the experimental chamber to clear the VC. Once it is safe to remove the animals, click on the OK button in the dialog box. The system will stop recording measurements to the file and the exposure is over.

NOTE: If a manual system is used, the user must manually turn off VC flow at the end of the exposure duration and time for VC clearance at the end of exposure must be calculated.

\section{Post-exposure}

1. Turn stopcock on the valve for the VC gas tank to the closed position and turn off all airflows in the exposure chamber. Turn the rotameter until no airflow is flowing through the control chamber.

2. Remove the doors from each chamber to provide ventilation to the mice. Remove the cage racks from the chambers. Under a hood, remove the mice from their exposure cages and place them back into their housing cages. Transport all mice back into their housing room for overnight housing in regular cages.

3. Dispose of any waste from the excreta pan into a Department of Environmental Health \& Safety (DEHS)-approved biohazard container, as these may be considered a chemical hazard by institutional environmental health services. Clean the chamber doors, excreta pan, exposure cage rack and exposure chamber for the experimental and control systems.

\section{Validation of VC concentration within chambers during exposure}

1. Conduct a measurement of the $\mathrm{VC}$ concentration within the experimental chamber halfway through each exposure ( $3 \mathrm{~h}$ ).

2. Break the glass tips on a VC detector tube and a pretreat tube. Attach the flow-out end of the VC detector tube to the detector tube pump. Attach the flow-in end of the VC detector tube to the flow-out end of the pretreat tube with a short piece of tubing. Attach a short piece of tubing to the flow-in end of the pretreat tube.

3. Remove a plug from one of the sampling ports that is near the breathing zone of the mice. Attach the tubing from the flow-in end of the pretreat tube to the sampling port.

4. From the full in position, extend the handle on the piston of the detector tube pump to the full out position. This will pull $100 \mathrm{~mL}$ of sampled gas from the chamber into the VC detector tube over a period of $90 \mathrm{~s}$. After waiting the $90 \mathrm{~s}$, push the handle back in.

5. Repeat step 7.4 three more times so that a total of $400 \mathrm{~mL}$ is pulled into the VC detector tube.

6. Remove the tube from the sampling port of the chamber and reinsert the plug into the port. Inspect the color change of the $V C$ detector tube to ascertain the VC concentration within the chamber. 
7. Record the VC detector tube reading in the laboratory notebook and compare to the theoretical value. Dispose of the VC detector tube and pretreat tube in a suitable container.

\section{Termination of inhalation exposure experiment}

NOTE: After desired timepoint of exposure, for example, 6,8 , and/or 12 weeks after beginning of inhalation exposure, the experiments are being terminated and animals will be euthanized (see Figure 1 for timeline).

1. Fast the mice $4 \mathrm{~h}$ prior to the time of euthanasia. NOTE: This procedure allows determination of fasting blood glucose and insulin levels for metabolic analysis.

2. Use a euthanasia approach consistent with the American Veterinary Medical Association (AVMA) guidelines, such as anesthesia followed by exsanguination.

3. Administer ketamine/xylazine $(100 / 15 \mathrm{mg} / \mathrm{kg})$ by intraperitoneal injection to each mouse to induce anesthesia. NOTE: Avoid sodium pentobarbital as a pre-euthanasia anesthetic, as vinyl chloride exposure may impede its effectiveness.

4. Collect blood from the inferior vena cava into sodium citrate solution (final, $0.38 \%$ ), to prevent blood coagulation and for sample preservation.

5. Remove the liver and/or any other desired organ. Dissect the liver and snap-freeze portions in liquid nitrogen, embed in frozen specimen medium, and fix in $10 \%$ buffered formalin for histology.

6. Separate plasma from blood via centrifugation and transfer the citrated plasma into a suitable tube and store at $-80^{\circ} \mathrm{C}$ until needed for analysis.

7. To evaluate histological indices of liver injury, perform hematoxylin and eosin (H\&E) staining with $5 \mu \mathrm{M}$ formalin fixed-paraffin embedded liver sections and obtain images with a brightfield microscope.

8. To obtain plasma transaminase levels, perform both alanine aminotransferase (ALT) and aspartate aminotransferase (AST) kinetic assays on the citrated plasma using commercially available kits.

NOTE: For quality control, plasma transaminases for C57BI/6J mice should be in the normal range (35-45 IU/L) for the LFD+VC group, while values should be elevated $(\sim 150 \mathrm{IU} / \mathrm{L})$ for the HFD+VC group (Figure $3 \mathrm{C}$ ).

\section{Representative Results}

Over the course of the experiment, animal body weight and food consumption were monitored weekly to ensure animal health and evaluate in vivo metabolism. Figure 3A depicts body weight and food consumption for a 12 week experiment. Body weight was measured once per week and food consumption was measured twice per week for all groups. All mice gained weight throughout the course of the study. While, as expected the mice in the HFD groups gained more weight as the mice in the LFD groups, the mice exposed to VC did not gain more weight than the mice in the respective control group. Food consumption was not different between all groups ${ }^{12,24}$.

Figure 3B depicts representative photomicrographs of liver sections stained with H\&E for analysis of general morphology. In the LFD group, VC caused no overt pathologic changes. HFD feeding significantly increased steatosis (fat accumulation) and VC exposure increased this effect. Moreover, VC exposure in the HFD group resulted in some inflammatory foci ${ }^{12,24}$.

Plasma transaminase (ALT and AST) levels were measured as indicators of liver damage and an elevated transaminase level is an indicator of liver damage. In the LFD group, VC did not increase transaminase levels. HFD alone slightly increased transaminase levels and importantly VC significantly enhanced this effect (Figure $3 \mathbf{C})^{12,24}$.

Liver weight to body weight ratios were calculated for each group. HFD significantly increased the liver to body weight ratios. However, VC did not significantly increase this effect (Figure 3D) ${ }^{12}$.

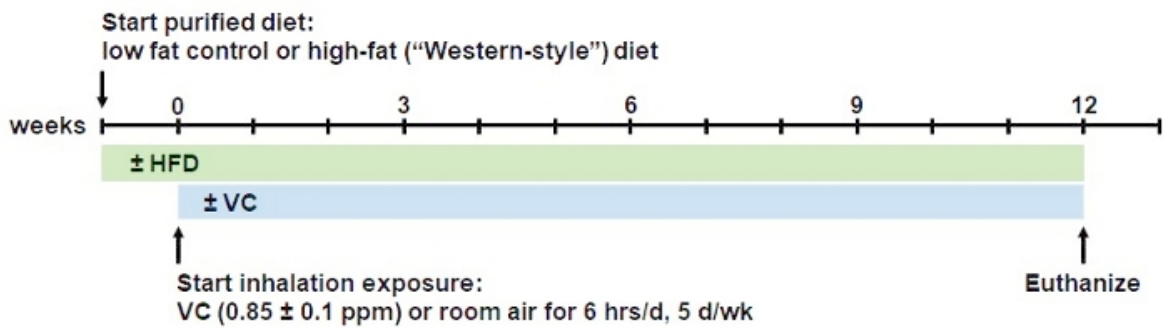

Figure 1: Overview of the inhalation model procedure. Mice are fed the respective low fat (13\% saturated fat) or high-fat (42\% saturated fat) diets ad libitum for 1 week to acclimatize them to the purified diet. After one week, mice are introduced to the inhalation regimen. For that, mice are placed in state-of-the-art whole-body inhalation chambers for exposure to a sub-OSHA level VC concentration of $<1 \mathrm{ppm}(0.85 \mathrm{ppm} \pm 0.1$ $\mathrm{ppm}$ ) or room air (control) for $6 \mathrm{~h} /$ day, 5 days/week, for 12 weeks. During the inhalation procedure the mice are allowed free access to food and water. At 12 weeks, mice are euthanized in the morning. This model can be extended to longer periods of chronic exposure. Please click here to view a larger version of this figure. 


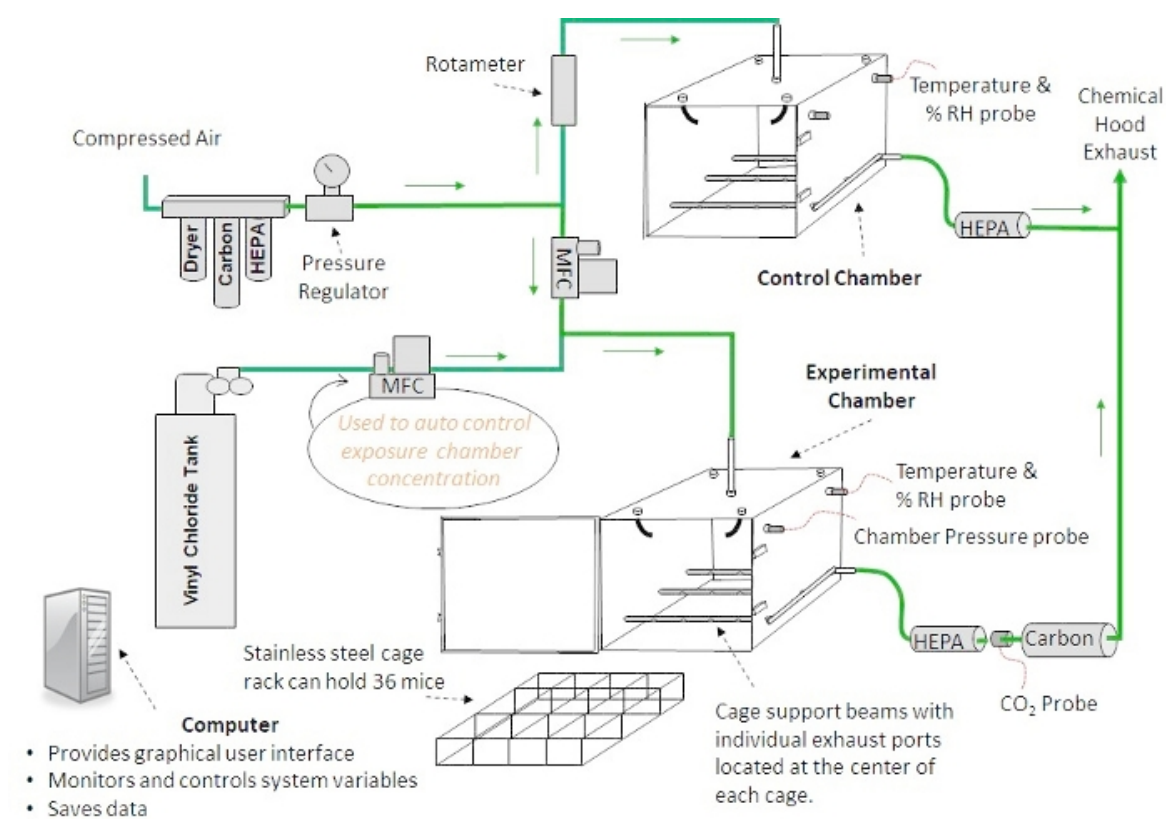

Figure 2: Inhalation chamber design. A diagram of an automated inhalation exposure system that provides homogenous toxicant concentrations is shown. Custom software allows the user to change, monitor and record environmental variables during inhalation exposures. Please click here to view a larger version of this figure.

A
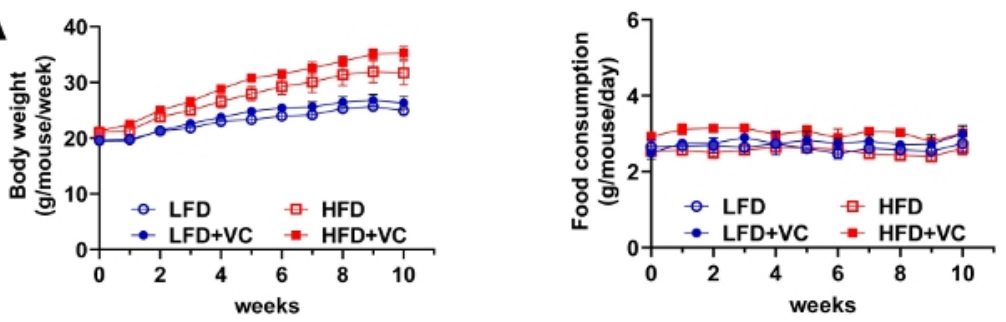

B
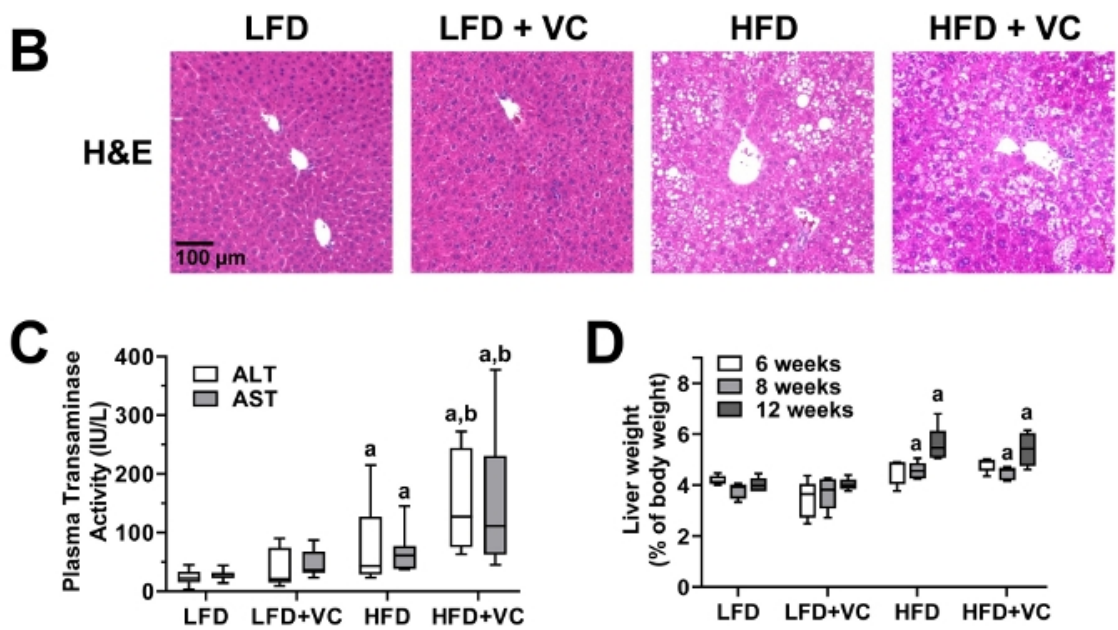

Figure 3: Vinyl chloride alone does not cause overt liver injury but enhances diet-induced liver disease. (A) Body weight and food consumption were monitored weekly. (B) Representative photomicrographs of general liver morphology by H\&E staining are shown (magnification $=200 x$ ). (C) Citrated plasma was collected at the end of the exposure period and analyzed for transaminase enzymatic activity as an index of liver damage. (D) Liver weight was determined at different experimental time points and compared to whole body weight. Results are presented as the mean \pm SEM. ${ }^{a}, p<0.05$ compared to respective LFD control; ${ }^{b}, p<0.05$ compared to absence of VC. Samples size per group $n$ $=8-10$. Please click here to view a larger version of this figure.

\section{Discussion}

This model of VC-enhanced NAFLD is a novel method to evaluate the effect of sub-OSHA limit VC exposure in a whole-body inhalation paradigm. This model allows investigators to study the sub-hepatotoxic and sensitizing effects by low levels of VC alone. Indeed, this co- 
exposure model achieves enhanced liver injury, elevation of plasma ALT and AST and moderate inflammation, while largely not affecting other organ systems, such as heart, at this concentration ${ }^{23}$. This chronic model requires whole-body inhalation chambers but minimizes stress and exposure concentrations. Although the protocol presented here is a software-driven approach, our experience has shown that the manual approach is also an accurate and consistent method of exposure ${ }^{12,24}$. Moreover, it is easily applicable to multiple research areas including other organ damage $e^{23}$ caused by volatile organic compound exposure ${ }^{22}$. Notably, this model may more closely resemble the pathogenesis of human co-exposures to environmental chemicals and underlying disease ${ }^{5}$.

In order to obtain similar results, certain critical steps of protocol optimization must be achieved. For example, investigators must establish that the concentration of VC or other toxicant within the chambers is within the desired range of exposure (i.e., low-level, sub-OSHA, or acute levels). Optimizing this step of the inhalation chamber is critical for a successful model of the human exposure of interest. Second, adjusting the time of exposure per day and duration of experiment may also be modified. Per the interests of this group, an occupational exposure setting was achieved, and an additional parameter of diet was also studied. However, environmental and acute exposures may also be modeled with this protocol.

This work challenges the paradigm that current exposure limits of VC (occupational and environmental) are safe. Indeed, although the current OSHA exposure limit for $\mathrm{VC}$ is $1 \mathrm{ppm}$, this model has proven that concentrations of $\mathrm{VC}$ below this limit are sufficient to enhance liver injury caused by HFD in mice. This protocol allows investigators to study and characterize a novel toxicant exposure paradigm and to model TASH.

This is the first model of chronic, low-dose VC exposure. Previous work used very high bolus concentrations, acute exposures or active metabolites as surrogates for VC exposure. All of these approaches decrease the relevance of the findings to human exposure. Therefore, this novel model of TASH-NAFLD interaction provides the necessary platform for investigators to examine complex interactions of low-level VC exposure.

This model of toxicant-induced liver injury can be used for other volatile organic compounds and also to study other interactions that may impact the liver and other organ systems $\mathrm{s}^{8,22,23}$. Moreover, this model has been, and can be further, used to investigate intervention therapies and indepth mechanistic studies of the mode of action for this prevalent toxicant ${ }^{24}$. As VC is a known carcinogen ${ }^{26,27,28}$, this exposure paradigm can also be modified for the study of VC-induced cancer. Other co-morbidities like alcoholic liver disease may also be enhanced by VC co-exposure. Additionally, it would be of interest to study different types of fat, such as polyunsaturated fat ${ }^{18,29,30}$, or different types of carbohydrates ${ }^{31}$ and their co-exposure with VC in this model. Indeed, all of these factors are known to have differential effects on the development of liver injury and may play a role in $\mathrm{VC}$-induced hepatic disease.

In conclusion, this is a novel inhalation model of environmental toxicant-induced liver injury and establishes an exposure paradigm for chronic, low-level VC exposure. The concentration of VC used in this model is sub-hepatotoxic by itself, while it enhances liver injury caused by another factor (HFD) in mice. This model will allow investigators to study mechanisms and interventions for chronic VC toxicity and may be helpful for translational studies looking at exposed human subjects and at the highest risk for exposure.

\section{Disclosures}

WT Goldsmith has financial interest in IEStechno, which is the template for the system described. The remaining authors have nothing to disclose.

\section{Acknowledgments}

This study was funded by awards from the National Institutes of Health (K01 DK096042 and R03 DK107912) to Juliane Beier. Research was also supported by an Institutional Development Award (IDeA) from the National Institute of General Medical Sciences of the National Institutes of Health under grant number P20GM113226 and the National Institute on Alcohol Abuse and Alcoholism of the National Institutes of Health under Award Number P50AA024337. The content is solely the responsibility of the authors and does not necessarily represent the official views of the National Institutes of Health.

\section{References}

1. Sass, J. B., Castleman, B., Wallinga, D. Vinyl chloride: a case study of data suppression and misrepresentation. Environmental Health Perspectives. 113 (7), 809-812 (2005).

2. ATSDR. Agency for Toxic Substances and Disease Registry (ATSDR): Toxicological profile for Vinyl Chloride. (2006).

3. Wahlang, B. et al. Toxicant-associated steatohepatitis. Toxicologic Pathology. 41 (2), 343-360 (2013).

4. Cave, M. et al. Toxicant-associated steatohepatitis in vinyl chloride workers. Hepatology. 51 (2), 474-481 (2010).

5. Cave, M., Falkner, K. C., McClain, C. J. Occupational and Environmental Hepatotoxicity. In Zakim and Boyer's Hepatology. Edited by Boyer, D. T., Manns, M. P., Sanyal, A. J., 476-492, Saunders. Philadelphia, PA (2012).

6. Tamburro, C. H., Makk, L., Popper, H. Early hepatic histologic alterations among chemical (vinyl monomer) workers. Hepatology. 4 (3), 413-418 (1984).

7. EPA. Toxicological review of vinyl chloride in support of summary information on the Integrated Risk Information System (IRIS). (2000).

8. Lang, A. L., Beier, J. I. Interaction of volatile organic compounds and underlying liver disease: a new paradigm for risk. Biological Chemistry. 399 (11), 1237-1248 (2018).

9. Abplanalp, W. et al. Benzene exposure is associated with cardiovascular disease risk. PLoS ONE. 12 (9), e0183602 (2017).

10. Younossi, Z. et al. Global burden of NAFLD and NASH: trends, predictions, risk factors and prevention. Nature Reviews Gastroenterology \& Hepatology. 15 (1), 11-20 (2018).

11. Younossi, Z. M. Non-alcoholic fatty liver disease - A global public health perspective. Journal of Hepatology. 70 (3), $531-544$ (2019). 
12. Lang, A. L. et al. Vinyl chloride dysregulates metabolic homeostasis and enhances diet-induced liver injury in mice. Hepatology Communications. 2 (3), 270-284 (2018).

13. Lotti, M. Do occupational exposures to vinyl chloride cause hepatocellular carcinoma and cirrhosis? Liver International. 37 (5), $630-633$ (2017).

14. Antweiler, H. Studies on the metabolism of vinyl chloride. Environmental Health Perspectives. 17, 217-219 (1976).

15. Bolt, H. M. Metabolic activation of vinyl chloride, formation of nucleic acid adducts and relevance to carcinogenesis. IARC Scientific Publications. (70), 261-268 (1986).

16. Guengerich, F. P., Crawford, W. M., Jr., Watanabe, P. G. Activation of vinyl chloride to covalently bound metabolites: roles of 2-chloroethylene oxide and 2-chloroacetaldehyde. Biochemistry. 18 (23), 5177-5182 (1979).

17. Anders, L. C. et al. Vinyl Chloride Metabolites Potentiate Inflammatory Liver Injury Caused by LPS in Mice. Toxicological Sciences. 151 (2), 312-323 (2016).

18. Anders, L. C. et al. Role of dietary fatty acids in liver injury caused by vinyl chloride metabolites in mice. Toxicology and Applied Pharmacology. 311, 34-41 (2016).

19. Morinello, E. J., Koc, H., Ranasinghe, A., Swenberg, J. A. Differential induction of N(2),3-ethenoguanine in rat brain and liver after exposure to vinyl chloride. Cancer Research. 62 (18), 5183-5188 (2002).

20. Guardiola, J. J. et al. Occupational exposures at a polyvinyl chloride production facility are associated with significant changes to the plasma metabolome. Toxicology and Applied Pharmacology. 313, 47-56 (2016).

21. Chen, L. C., Lippmann, M. Inhalation toxicology methods: the generation and characterization of exposure atmospheres and inhalational exposures. Current Protocols in Toxicology. 63 (1), 24.4.1-24.4.23 (2015).

22. Wahlang, B. et al. Mechanisms of Environmental Contributions to Fatty Liver Disease. Current Environmental Health Reports. 6 (3), 80-94 (2019).

23. Liang, Y. et al. Exposure to Vinyl Chloride and Its Influence on Western Diet-Induced Cardiac Remodeling. Chemical Research in Toxicology. 31 (6), 482-493 (2018).

24. Chen, L., Lang, A. L., Poff, G. D., Ding, W. X., Beier, J. I. Vinyl chloride-induced interaction of nonalcoholic and toxicant-associated steatohepatitis: Protection by the ALDH2 activator Alda-1. Redox Biology. 24, 101205 (2019).

25. Goldsmith, W. T. et al. A computer-controlled whole-body inhalation exposure system for the oil dispersant COREXIT EC9500A. Journal of Toxicology and Environmental Health. Part A. 74 (21), 1368-1380 (2011).

26. IARC Working Group on the Evaluation of Carcinogenic Risk to Humans. IARC Monographs on the Evaluation of Carcinogenic Risks to Humans. International Agency for Research on Cancer. Lyon, France (2008).

27. IARC. Chemical agents and related occupations. IARC Monographs on the Evaluation of Carcinogenic Risks to Humans. 100 (Pt F), 9-562 (2012).

28. Fedeli, U. et al. Mortality from liver angiosarcoma, hepatocellular carcinoma, and cirrhosis among vinyl chloride workers. American Journal of Industrial Medicine. 62 (1), 14-20 (2019).

29. Kirpich, I. A. et al. Ethanol and dietary unsaturated fat (corn oil/linoleic acid enriched) cause intestinal inflammation and impaired intestinal barrier defense in mice chronically fed alcohol. Alcohol. 47 (3), 257-264 (2013).

30. Kirpich, I. A. et al. Saturated and Unsaturated Dietary Fats Differentially Modulate Ethanol-Induced Changes in Gut Microbiome and Metabolome in a Mouse Model of Alcoholic Liver Disease. American Journal of Pathology. 186 (4), 765-776 (2016).

31. Spruss, A., Bergheim, I. Dietary fructose and intestinal barrier: potential risk factor in the pathogenesis of nonalcoholic fatty liver disease. Journal of Nutritional Biochemistry. 20 (9), 657-662 (2009). 